\title{
Wake patterns of freely vibrating side-by-side circular cylinders in laminar flows
}

\author{
Weilin Chen ${ }^{1}$, Chunning Ji ${ }^{1,2, *}$, Dong Xu ${ }^{1}$, Narakorn Srinil ${ }^{3}$ \\ (1. State Key Laboratory of Hydraulic Engineering Simulation and Safety, Tianjin University, \\ Tianjin 300072, China. \\ 2. Collaborative Innovation Centre for Advanced Ship and Deep-Sea Exploration, Shanghai \\ 200240, China. \\ 3. School of Engineering, Newcastle University, NE1 7RU, Newcastle upon Tyne, United \\ Kingdom.)
}

\begin{abstract}
Wake patterns behind two freely vibrating circular cylinders in side-by-side arrangements are numerically investigated through two-dimensional computational fluid dynamics simulations. The two cylinders, with an equal diameter and a low mass ratio (the cylinder mass/the displaced fluid mass) of 2, can only oscillate in the transverse or cross-flow direction, and they are subject to the uniform laminar flows with the Reynolds number of 100 . The center-to-center spacing over a cylinder diameter ratio $(s / D)$ is varied from 2 to 5 . For each $s / D$, the reduced velocity $U_{r}$ (defined by $U_{\infty} / f_{n} D$, where $U_{\infty}$ is the free-stream velocity and $f_{n}$ is the cylinder natural frequency) is varied from 0 to 30. A small parametric increment of $\Delta U_{r}=0.1$ and $\Delta s / D=0.1$ is considered, providing a comprehensive analysis and insights into the wake patterns of the vortex shedding behind both cylinders. With more than 1500 new simulation cases, 8 distinctive wake patterns are identified through a map of $U_{r}$ and $s / D$, with several well-defined boundaries governing different flow patterns. Qualitatively distinctive wake characteristics are analyzed through the evaluations of vortex dynamics, hydrodynamic lift/drag forces and cylinder vibration responses.
\end{abstract}

Keywords: vortex-induced vibration, circular cylinder, side-by-side arrangement, wake pattern, fluid-structure interaction, computational fluid dynamics

\section{Introduction}

Insights into fluid wakes are crucial for predicting the vibration responses and understanding the underlying physics of flows past cylindrical bluff bodies. Wake patterns exhibit distinctive features in the cases of single versus multiple bodies placed in flows. Recent reviews on wakes behind cylindrical structures can be found in Zdravkovich (1997), Williamson and Govardhan (2004), Sumner (2010), and Zhou and Alam (2016). In comparison with the wakes behind an isolated stationary/vibrating cylinder or multiple stationary cylinders, the wakes trailing the multiple vibrating cylinders are much less understood. In the present paper, we aim to explore the wake patterns of flows past two freely vibrating circular cylinders in a side-by-side arrangement by focusing on the two fundamental effects of center-to-center spacing ratio and reduced velocity.

${ }^{*}$ Corresponding author: cnji@tju.edu.cn 
For flows past two circular cylinders, the interference between the cylinders may be classified into proximity interference, wake interference, and a combination of both (Zdravkovich, 1977), according to the spacing between the cylinders and the orientation relative to the free stream. The proximity interference occurs when two staggered, or side-by-side, cylinders are close enough, or when the tandem downstream cylinder is entirely embraced by the shear layers separated from the upstream cylinder. The wake interference occurs when the two tandem cylinders are relatively far from each other, such that the fully developed vortices shed from the upstream cylinder are present in the gap between the two cylinders. This feature also exists in the staggered arrangement with a small transverse spacing and a large streamwise spacing. The combined interference case can be found in an overlapping region between the proximity and wake interferences.

In the side-by-side arrangement, three flow regimes are found at different normalized spacing ratios $(s / D)$ (Sumner, 2010). When the spacing ratio is small $(s / D \leq 1.1-1.2)$, the gap flow between the cylinders is rather weak without a vortex-shedding. Because vortices are only alternately shed from the free-stream sides of the two cylinders (Sumner et al., 1999), this is named as a single bluffbody flow regime. When the spacing becomes large ( $s / D \geq 2.2-2.5)$, a synchronized vortex shedding occurs. Parallel vortex streets shed from the cylinders are in- or anti-phase synchronized, depending on $s / D$ and the Reynolds number (Re) (Bearman and Wadcock, 1973; Williamson, 1985). At an intermediate spacing (1.1-1.2 $<s / D<2.2-2.5)$, the gap flow is biased stably or alternately to one of the cylinders, leading to a narrow-wide wake pattern. The cylinder with the narrow wake, compared to its counterpart, has a higher vortex-shedding frequency and a larger drag force (Bearman and Wadcock, 1973; Kim and Durbin, 1988). The alternately biased gap flow is also named as the flipflopping flow (Bearman and Wadcock, 1973) in which the gap flow changes its direction occasionally and randomly. The flip-over time interval of the gap flow is closely related to $s / D$ and Re (Kim and Durbin,1988), from several orders of the magnitude greater than the vortex shedding period in turbulent flows to a few vortex shedding periods in laminar flows. Particularly, in low-Re cases, several well-defined wake patterns, such as the anti-phase, in-phase, flip-flopping, deflected, single bluff-body and steady patterns have been reported (Kang, 2003; Liu et al., 2007; Supradeepan and Roy, 2014).

Freely or forced vibrating cylinders further intensify the vortex shedding, thus leading to a more complicated wake pattern compared to the stationary cases. To the best of the authors' knowledge, a refined and comprehensive overview of the wake patterns has not yet been achieved. Xu et al. (2008) found that vibrations of the two cylinders $(\operatorname{Re}=200,1.2 \leq s / D \leq 3.2)$ significantly affect the wake patterns, and a synchronized state can be achieved only when the amplitude is sufficiently large. Cui et al. (2014) observed from numerical simulations with two side-by-side elastically coupled cylinders $(\operatorname{Re}=5000, s / D=3.0)$ that, when the amplitude is small, the interaction between the cylinders is weak, and the wake pattern exhibits a dual-2S mode. Liu et al. (2001) confirmed the critical $s / D=3.0(\operatorname{Re}=200)$; for a larger $s / D$, the neighboring cylinder does not show a noteworthy effect on the vibration response. This spacing is also a critical value for the anti-phase 
wake. Recently, Chen et al. (2015a) summarized six distinctive wake patterns $(\operatorname{Re}=100,2.0 \leq s / D$ $\leq 5.0)$ including the irregular, in-phase and out-of-phase flip-flopping, in-phase and anti-phase synchronized, and biased anti-phase synchronized patterns. Chen et al. (2015b) further studied the hybrid wake pattern in the vortex-induced vibration (VIV) of two side-by-side cylinders ( $R e=80$ $150,2.0 \leq s / D \leq 5.0)$.

In this paper, we characterize and present the wake patterns of two freely vibrating side-byside circular cylinders, aiming to provide a comprehensive overview of the wake patterns in the reduced velocity-cylinder spacing $\left(U_{r}, S / D\right)$ map within a large parametric range, and to identify the refined boundaries of flow regimes by employing small parametric increments. The remaining paper is organized as follows. In Section 2, a numerical methodology and validation cases are presented. Features of wake patterns, hydrodynamic drag/lift forces, and cylinder vibrations are investigated and discussed in Section 3. Key conclusions of new findings are summarized in Section 4.

\section{Numerical Methodology}

\subsection{Fluid-structure interaction model}

The equations governing the fluid flows are the incompressible Navier-Stokes and continuity equations. The two-step predictor-corrector procedure is adopted for decoupling these equations. The resultant pressure Poisson equation is solved by using the BiCGSTAB scheme and the geometric multi-grid method. The second-order Adams-Bashforth time marching scheme is employed to calculate a new velocity field.

The dynamics of the two elastically-mounted cylinders is simplified as a linear mass-damperspring system. The equations of cylinder motions are based on the Newton's second law, which are solved by applying a standard Newmark- $\beta$ method with a second-order temporal accuracy.

The fluid-structure interaction (FSI) is simulated by using the Immersed Boundary (IB) method which was first introduced by Peskin (1972) in simulating blood flows around a flexible leaflet of a human heart. In the IB framework, the flow governing equations are discretized on a fixed Cartesian grid which generally does not conform to the geometry of the moving solids. As a result, the boundary conditions on the fluid-cylinder interface, manifesting the interaction between the flow and the structure, cannot be imposed directly. Instead, an extra body force is added to the momentum equation by using the interpolation and distribution functions to take such FSI into account. Compared with the conventional numerical methods, the IB method has some key advantages, particularly in FSI simulations with topological changes. Another merit of the IB method lies in its parameterized and fast implementation for a large number of simulations with different geometric configurations, in comparison with conventional methods using the body-conformal grids. For the sake of conciseness, details of the methodology can be referred to our previous works (Ji et al., 2012; Chen et al., 2015a) to attain further information.

In two-dimensional simulations, the streamwise and transverse lengths of the computational 


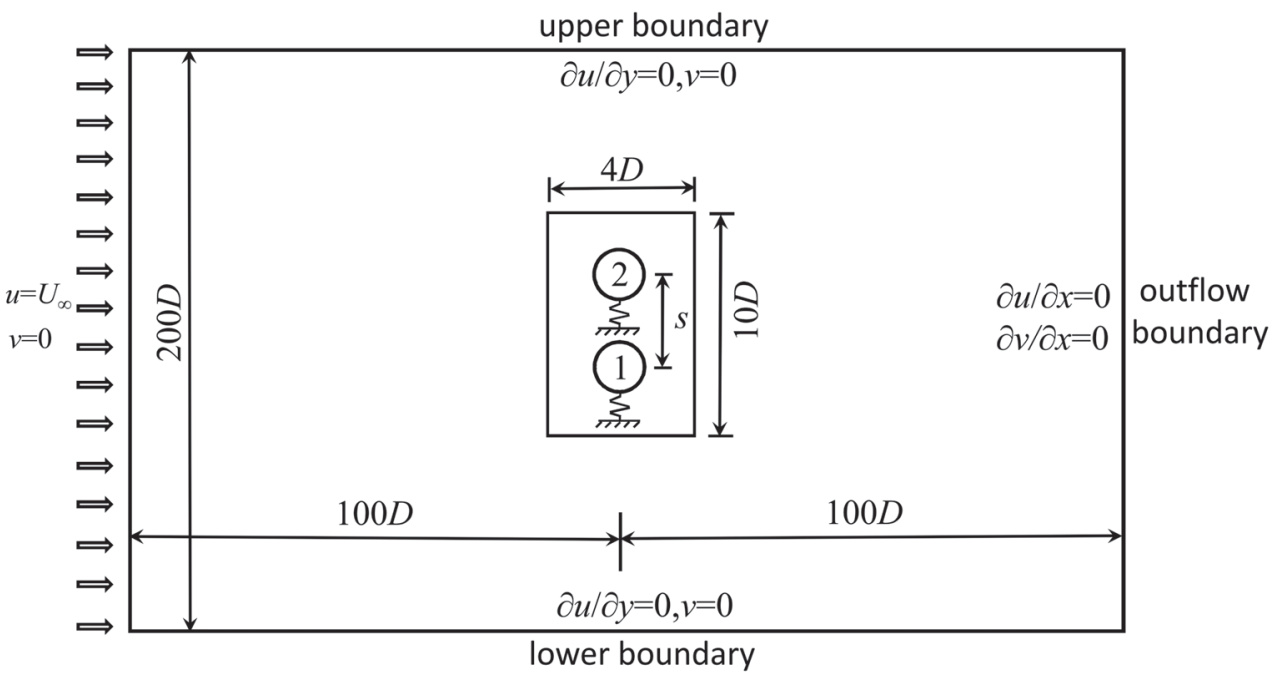

Figure 1. Computational domain of flows past two side-by-side oscillating cylinders

As for the simulation parameters, the center-to-center spacing ratio range is $2.0 \leq s / D \leq 5.0$, the reduced velocity range is $0 \leq U_{r}=U_{\infty} / f_{n} D \leq 30$, the Reynolds number is $\operatorname{Re}=U_{\infty} D / v=100$, the mass ratio is $m^{*}=4 m / \pi \rho D^{2}=2.0$, and the structural damping ratio is set to be zero for the purpose of exciting largest vibration amplitudes. $U_{\infty}$ is the unaffected free-stream velocity, $v$ the fluid kinematic viscosity, $\rho$ the fluid density, $m$ the cylinder mass, and $f_{n}$ the cylinder natural frequency. Both cylinders can only vibrate in the transverse direction. In-line motion will be accounted for in future studies.

\subsection{Model validation}

VIV of an isolated circular cylinder is used as a benchmark case to validate the present numerical method. The isolated cylinder with $m^{*}=10$ freely oscillates in both cross-flow and inline directions. Re is $100, U_{r}$ is 6.0 , and the computational domain is $[-25 D, 25.5 D] \times[-10 D, 10 D]$. The same parameters were applied in numerical simulations of Prasanth and Mittal (2008). Table 1 shows that the present numerical results agree quite well with those of Prasanth and Mittal (2008), with the maximum difference being less than $2 \%$. This highlights a high accuracy and fidelity of the present IB method. More validation cases can be found in our previous works (Chen et al., 2015a; 2015b; 2018). 
The adopted non-dimensional grid spacing is checked in the case of VIV of two side-by-side cylinders with $\operatorname{Re}=100, m^{*}=2.0, s / D=3.0$, and $U_{r}=5.0$. Table 2 shows a marginal difference $(<4 \%)$ between the results with $\Delta x / D(\Delta y / D)=1 / 64$ and $1 / 128$, indicating that the case of $\Delta x / D$ $(\Delta y / D)=1 / 64$ is grid-independent. It is worth noting that such mesh configuration was also applied in our previous studies (Chen et al., 2015a; 2015b; 2018), demonstrating an acceptable accuracy.

Table 1. VIV of an isolated cylinder with $\operatorname{Re}=100, U_{r}=6.0$, and $m^{*}=10 . S t$ is the Strouhal number, $Y_{\max } / D$ is the normalized maximum cross-flow vibration amplitude, $\bar{x} / D$ is the normalized mean inline displacement, $x_{r m s} / D$ is the normalized root-mean-square (r.m.s.) in-line vibration amplitude, $\bar{C}_{D}$ is the mean drag coefficient, $C_{D}^{\prime}$ is the r.m.s. drag coefficient, and $C_{L}^{\prime}$ is the r.m.s. lift coefficient.

\begin{tabular}{|c|c|c|c|c|c|c|c|}
\hline & $S t$ & $Y_{\max } / D$ & $\bar{x} / D$ & $x_{r m s} / D$ & $\bar{C}_{D}$ & $C_{D}^{\prime}$ & $C_{L}^{\prime}$ \\
\hline Present & 0.164 & 0.522 & 0.112 & 0.005 & 1.91 & 0.250 & 0.155 \\
\hline Prasanth and Mittal (2008)* & 0.164 & 0.516 & 0.111 & 0.005 & 1.90 & 0.255 & 0.157 \\
\hline Difference (\%) & 0 & 1.2 & 0.9 & 0 & 0.5 & 2.0 & 1.3 \\
\hline
\end{tabular}

${ }^{*}$ Direct numerical simulation results

Table 2. VIV of two side-by-side cylinders with $\operatorname{Re}=100, m^{*}=2.0, s / D=3.0$, and $U_{r}=5.0 . \bar{C}_{L}$ is the mean lift coefficient, and other symbols are defined as in Table 1. Values in the parentheses indicate a percent difference against results with $\Delta x / D(\Delta y / D)=1 / 128$.

\begin{tabular}{ccccccc}
\hline$\Delta x / D(\Delta y / D)$ & $\bar{C}_{D}$ & $C_{D}^{\prime}$ & $\bar{C}_{L}$ & $C_{L}^{\prime}$ & $Y_{\max } / D$ & $S t$ \\
\hline $1 / 32$ & $2.075(0.2)$ & $0.400(0.5)$ & $-0.248(5.5)$ & $0.403(19.6)$ & $0.556(1.8)$ & $0.179(0.6)$ \\
$1 / 64$ & $2.074(0.3)$ & $0.398(0)$ & $-0.243(3.4)$ & $0.350(3.7)$ & $0.545(0.2)$ & $0.180(0)$ \\
$1 / 128$ & 2.080 & 0.398 & -0.235 & 0.337 & 0.546 & 0.180 \\
\hline
\end{tabular}

\section{Numerical Results and Discussion}

To present a comprehensive wake pattern and partition map within a wide range of $0 \leq U_{r} \leq 30$ and $2 \leq s / D \leq 5$, very small increments of both the reduced velocity $\left(\Delta U_{r}=0.1\right)$ and the spacing ratio $(\Delta s / D=0.1)$ are adopted. More than 1500 simulations are carried out, each taking about 12 hours by using $362.9 \mathrm{GHz}$ CPU cores. Note that the case of $s / D<2$ is skipped to avoid a possible collision between the cylinders. Shown in Figure 2 is the wake partition in the $\left(U_{r}, s / D\right)$ parametric space. Eight distinct wake patterns can be identified, including the anti-phase synchronized pattern (AS), the in-phase synchronized pattern (IS), the biased anti-phase synchronized pattern (BAS), the in-phase flip-flopping pattern (IFF), the out-of-phase flip-flopping pattern (OFF), the long period flip-flopping pattern (LFF), the irregular pattern (IR) and the hybrid pattern (HB). Key features of these wake patterns are summarized in Table 3 based on subsequent parametric investigations. 


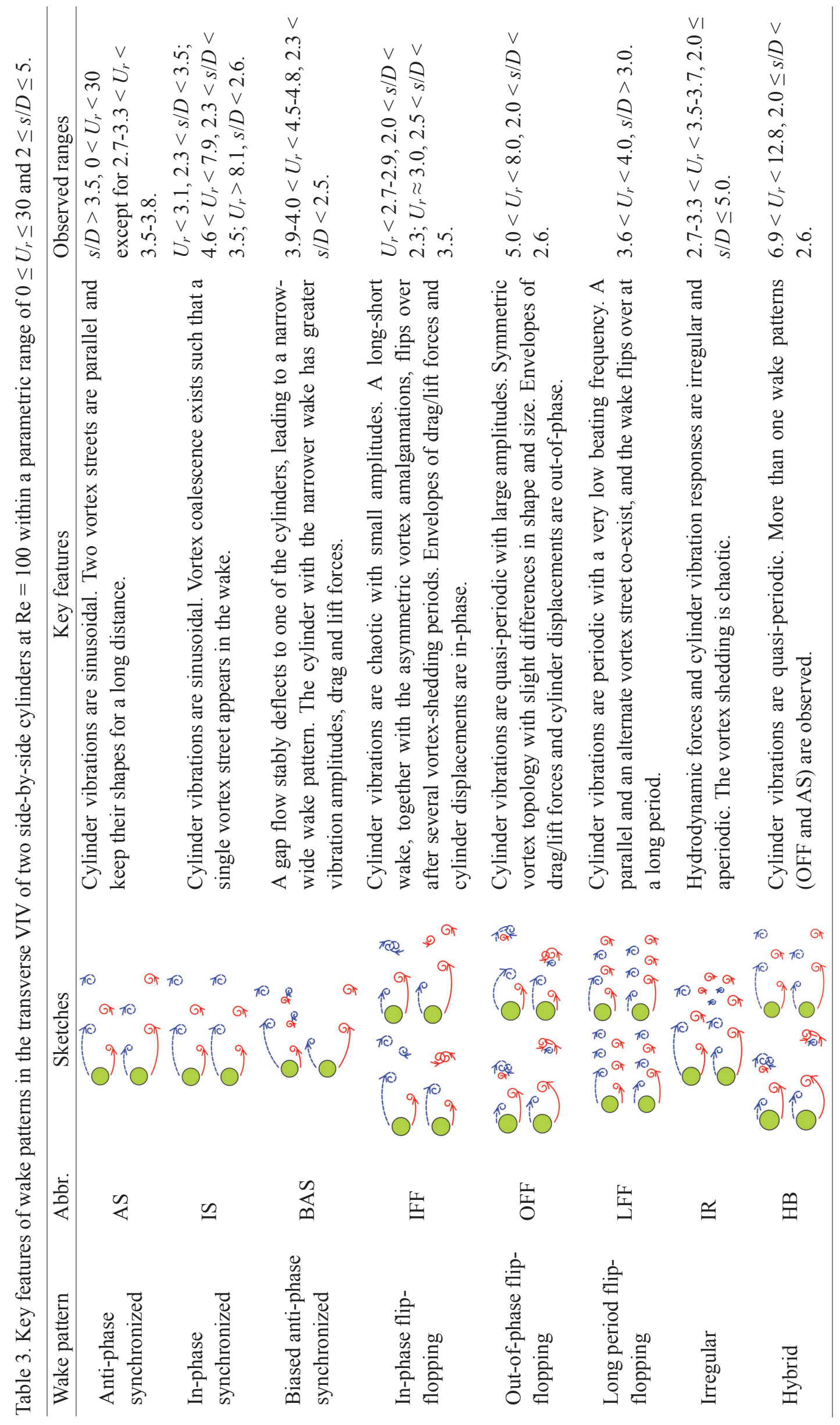

함 


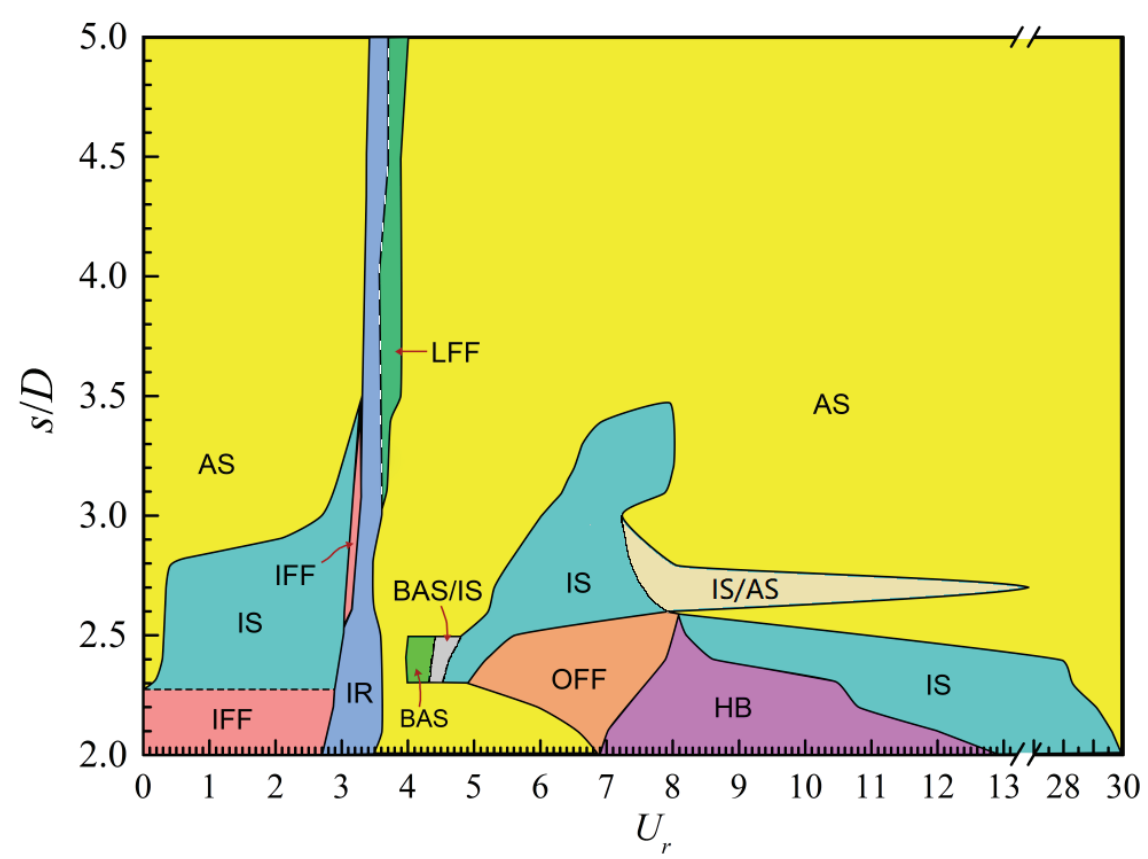

Figure 2. Wake regime and partition in the $\left(U_{r}, s / D\right)$ parametric space with $\operatorname{Re}=100$ and $m^{*}=2.0$ : the anti-phase synchronized pattern (AS), the in-phase synchronized pattern (IS), the biased antiphase synchronized pattern (BAS), the in-phase flip-flopping pattern (IFF), the out-of-phase flipflopping pattern (OFF), the long period flip-flopping pattern (LFF), the irregular pattern (IR) and the hybrid pattern (HB). BAS/IS and IS/AS indicate hysteresis wake patterns with respect to $U_{r}$.

Figure 3 illustrates the r.m.s vibration amplitudes (only for the lower cylinder) at four representative $s / D=2.2,2.8,3.5$ and 5 . These vibration curves appear to be similar despite the wake patterns with increasing $U_{r}$ are different. From Figures 2 and 3 , it is seen that in the desynchronized regions $\left(U_{r}<2.7-3.3\right.$ and $\left.U_{r}>8.0\right)$, where the vibration amplitudes are small, the wake sequentially experiences FF, IS and AS patterns with increasing $s / D$. It is worth explaining that IFF and OFF patterns belong to the flip-flopping (FF) pattern while the HB pattern is a combination of OFF and IS patterns. Thus, to some extent, the wake pattern in the desynchronized regions is the FF pattern at a small $s / D$. In the initial branch region $\left(2.7-3.3<U_{r}<3.5-3.8\right)$ with IR and LFF patterns, the latter one appears at a higher $s / D$. In this region, the wake is aperiodic and irregular, corresponding to a sharp increment of vibration amplitude. In the lower branch region (3.5-3.8 $\left.<U_{r}<8.0\right)$, all AS, IS, OFF and BAS patterns occur, with the AS pattern prevailing. It is interesting to note that there are two wake hysteresis regions: between BAS and IS patterns and between IS and AS patterns, existing at an intermediate $s / D$. With increasing or decreasing $U_{r}$, the wake shows the former or the latter pattern.

In summary, AS pattern occupies the top portion $(s / D>3.5)$ with an exception at 2.7-3.3 $<U_{r}$ $<3.5-3.8$ where IR and LFF patterns exist. IS pattern distributes in three sub-regions and takes the second largest portion in the $\left(U_{r}, s / D\right)$ plane. Other patterns spread in the lower part with $s / D<2.5$. Note that some of the boundaries, as indicated by the dashed lines, are unclear owing to the 
coexistence of the two adjacent patterns and the transitions: these make the boundaries very difficult to be clearly defined. Further, two hysteresis regions, i.e. BAS/IS and IS/AS, are identified in which the wake undergoes BAS (IS) and IS (AS) patterns when increasing (decreasing) $U_{r}$.

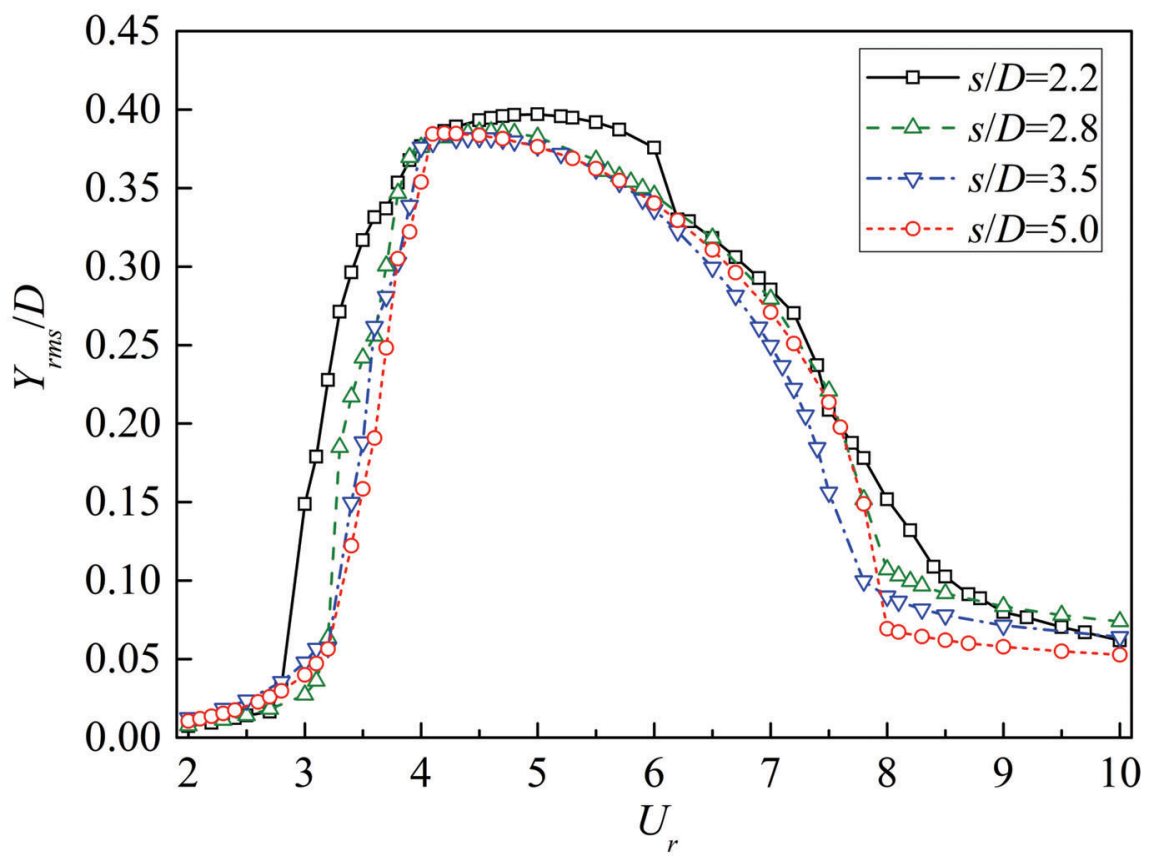

Figure 3. Vibration amplitudes of the lower cylinders at $s / D=2.2,2.8,3.5$ and 5 , all with $\operatorname{Re}=100$.

\subsection{Anti-phase synchronized pattern}

For AS pattern, the vortex-shedding behind the two cylinders is anti-phase, showing a dual-2S mode, see Figure 4(ai). Two parallel vortex streets in the wake keep their shapes for a long distance since no vortex coalescence takes place. However, as shown in Figure 4(aii), when $s / D$ is small, vortices shed from the inner shear layers of the cylinders are significantly weakened, compared with their free-stream side counterparts. On the other hand, the inner-side vortices dissipate quickly due to their relative closer distance. As a result, the inner-side vortex rows disappear in several vortex formation lengths. Without the segregating effects of the inner-side vortices, the free-stream side ones are stretched along the streamwise direction, gradually coalescing with upstream and downstream vortices in a sequence. As shown in Figure 4(aiii), a time history of the cylinder displacement is also in anti-phase, exhibiting a sinusoidal variation.

\subsection{In-phase synchronized pattern}

This pattern is displayed in Figure 4(bi) showing how the inner-side vortices break down into two parts, owing to a large-amplitude vibration of the cylinders. One of the parts merges with the free-stream side vortex shed from the other cylinder. The other part pairs with the amalgamated vortex shed in the previous half vortex-shedding cycle. Moreover, vortices consisting of the pairs have different vorticity, thus resulting in a $2 \mathrm{P}_{\mathrm{o}}$ mode. However, due to the weaker part of the pairs dissipating quickly, the wake transforms into a $2 \mathrm{~S}$ mode in a short distance downstream. When the 
vibration amplitude is small, the inner side vortex directly coalesces with the free-stream side vortex shed from the other cylinder, see Figure 4 (bii). In the downstream, the wake becomes similar to that in Figure 4 (bi). Shown in Figure 4(biii) is the displacement time history. Clearly, the two curves collapse, indicating a perfect phase alignment.

(ai)

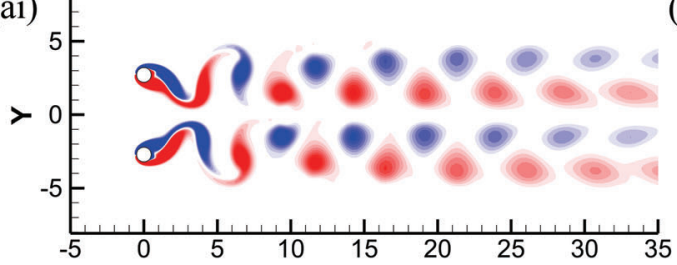

(aii)
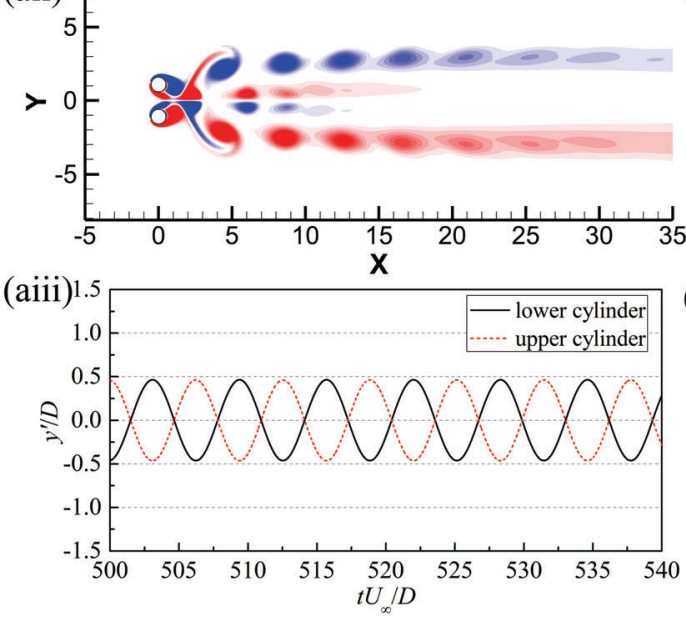

(bi)

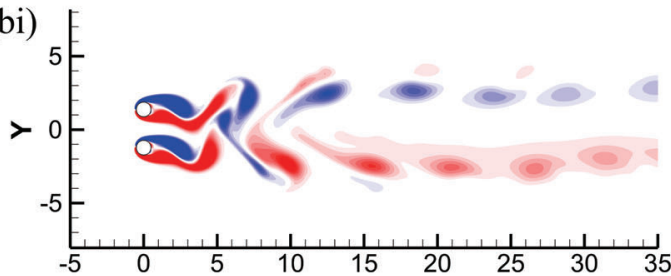

(bii)
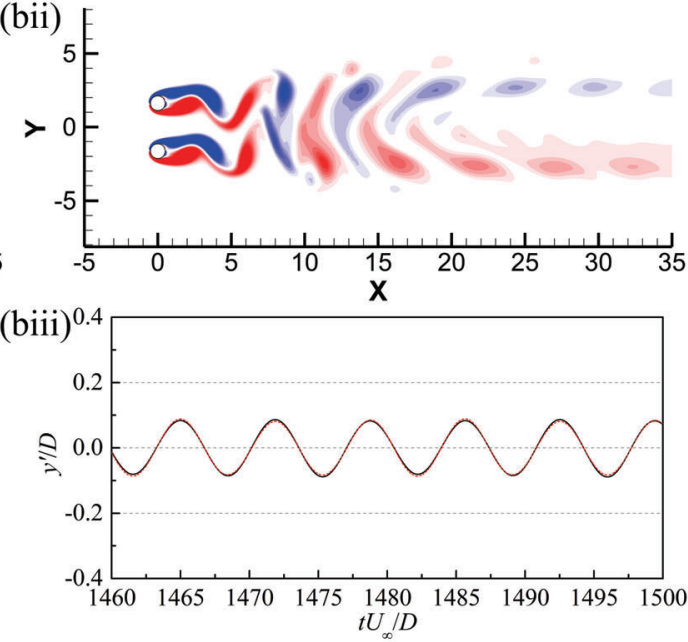

Figure 4. Wake patterns and time histories of VIV of two side-by-side circular cylinders. (ai) AS pattern at $U_{r}=6.2$ and $s / D=4.5$, (aii) AS pattern at $U_{r}=6.2$ and $s / D=2$, and (aiii) displacement histories at $U_{r}=6.2$ and $s / D=4.5$. (bi) IS pattern at $U_{r}=9.0$ and $s / D=2.4$, (bii) IS pattern at $U_{r}=$ 18.0 and $s / D=2.7$, and (biii) displacement histories at $U_{r}=9.0$ and $s / D=2.4$. Contours of the normalized Z-vorticity are in $[-1,1]$ with an increment of 0.125 (same for below figures).

\subsection{Biased anti-phase synchronized pattern}

This BAS pattern is observed in a small region of 3.9-4.0 $<U_{r}<4.5-4.8$ and $2.3<s / D<2.5$, as shown in Figure 2. In the range of 4.3-4.4 $<U_{r}<4.5-4.8$, the wake hysteresis exists in the BAS/IS region, i.e. the wake pattern is BAS or IS depending on either increasing or decreasing $U_{r}$. As depicted in Figure 5(a), vortices shed from the inner-side shear layers stably deflect towards the upper cylinder, which results in a narrow-wide wake pattern. The cylinder with the narrower wake has a larger vibration amplitude, drag and lift forces, as shown in Figure 5 (b-d). This is different from the observation in flows past two stationary side-by-side cylinders, in which the cylinder with the narrower wake experiences a larger drag but a smaller lift. From Figures 5(c) and 5(d), it is seen that the phase difference between the lift forces, and between the displacements of the cylinders, is about $123^{\circ}$, with the upper cylinder leading the lower one. This means that the inner-side vortex shed from the lower cylinder takes place slightly earlier than the inner-side vortex shed from the 
(a)
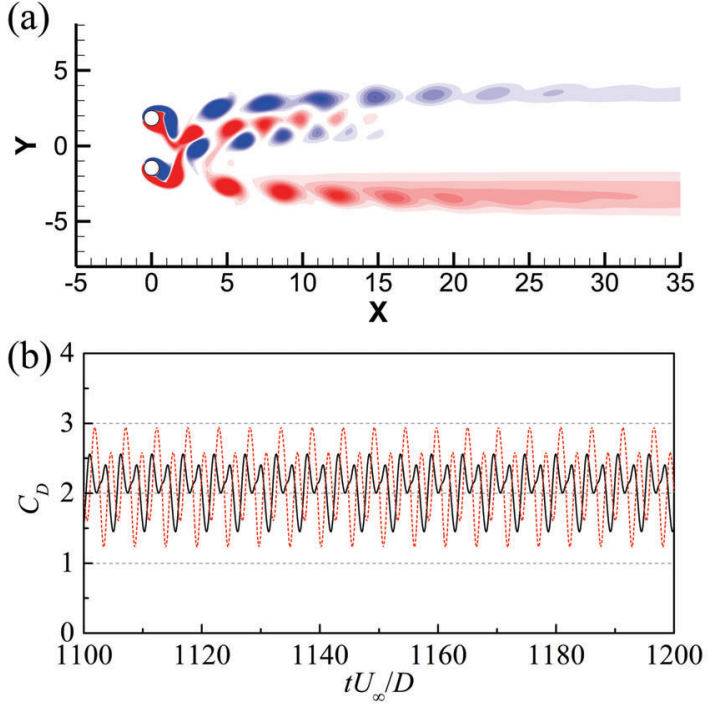

(c)

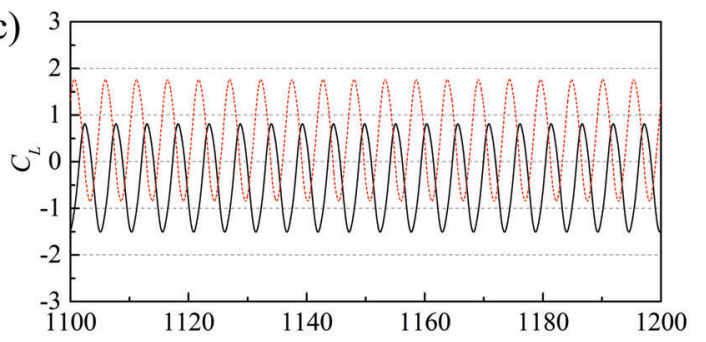

(d)

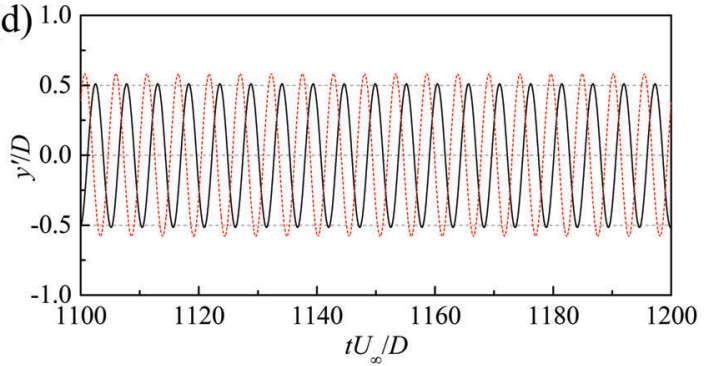

Figure 5. BAS pattern (a) and time histories of drag (b), lift (c) and displacement (d) for VIV of two side-by-side circular cylinders at $U_{r}=4.3$ and $s / D=2.4$.

\subsection{In-phase flip-flopping pattern}

This IFF pattern exists in a small region of $0<U_{r}<2.7-2.9$ and $2.0<s / D<2.3$ and a narrow stripe at $U_{r}=3.0$ with $2.5<s / D<3.5$, as shown in Figure 2. In these regions, the cylinders respond with small vibration amplitudes, as shown in Figure 3.

A flip-flopping vortex shedding is the most interesting phenomenon in VIV since it reflects a strong but unstable flow interference between two side-by-side cylinders. Kang (2003) found that the wake behind two side-by-side stationary cylinders has different widths. The vortex-shedding frequency and the drag on the cylinder with a narrow wake are larger than those on the cylinder with a wider wake. This narrow-wide wake flips over after several vortex-shedding periods. However, it is difficult to recognize the narrow-wide wake in VIV cases with vibrating cylinders due to the swinging gap flow (Chen et al. 2015a). Nonetheless, it reveals that the vortex formation length of one cylinder is obviously longer than the other one, with the flip-over action of this shortlong wake pattern happening quasi-periodically.

Figure 6 shows a time sequence of the vortex shedding within one vibration cycle by plotting the contours of the normalized Z-vorticity. Vortex A and Vortex B', which are shed from the gap side of the upper cylinder and the free-stream side of the lower cylinder, respectively, coalesce at a position about two vertex formation lengths downstream the cylinders. However, Vortex A' and 

and the gap side of the lower cylinder, respectively, do not merge at all. This can be attributed to the fact that the gap side shear layer of the lower cylinder is shorter, which makes the easier coalescence of Vortex A and Vortex B'. On the contrary, the longer gap side shear layer of the upper cylinder separates Vortex A' and Vortex B from each other, preventing the amalgamation of vortices.

Figure 7 displays time histories of the drag, lift and displacement, together with the wavelet transform (WT) and fast Fourier transform (FFT) analysis results at $U_{r}=2.0$ and $s / D=2.1$. In comparison with the vorticity contours in Figure 6 and the corresponding instantaneous drag, lift, and displacement marked by the dashed lines in Figure 7, it can be found that the lower cylinder with a shorter wake has a larger mean drag, fluctuating drag and lift, and vibration amplitude. The upper cylinder with a longer wake has a smaller mean drag, fluctuating drag and lift, and vibration amplitude. The larger fluid forces are caused by the closer vortices in the shorter wake to the cylinder's rear end, and vice versa. Moreover, the drag, lift and displacement exhibit the in-phase varying envelopes. In this particular case, amplitudes of the drag, lift and displacement achieve large values synchronously. Thus, the wake pattern is named as the in-phase flip-flopping. It should be noted that the in-phase flip-flopping vibration with small amplitudes appears to be random-like chaotic. This can be supported by the Poincaré maps of the two cylinders' trajectories shown in Figure 8(a). Herein, the "in-phase" does not imply the in-phase locked vibrations of the two cylinders as in IS pattern, but this definition indicates that temporal variations of the drag, lift and displacement envelopes are in-phase synchronized as illustrated in Figure 7.

A dominant lift frequency calculated by FFT is generally used as a direct measurement of the vortex-shedding frequency. It is worth noting that the spectral plot of the lift may have multiple peaks due to the wake-cylinder and cylinder-cylinder interactions. For IFF pattern, three meaningful peaks, together with multiple spikes, are observed, as shown in Figure 7(biv). The peak at $f_{l 3}=0.191$ corresponds to the vortex-shedding frequency of the short wake while the peak at $f_{12}=0.147$ represents the vortex-shedding frequency of the long wake. These indicate that the vortex shedding is faster in the shorter wake than in the longer one. This is straightforward to understand that the shorter shear layers are easier to roll up, thus having a higher vortex shedding frequency. The small peak at $f_{l 1}=0.044$ corresponds to the beating frequency of $f_{l 3}$ and $f_{l 2}$. This frequency also denotes the flip-flopping frequency of the short-long wake, as indicated in Chen et al. (2015a). The ratio ( $f_{13}$ $\left.+f_{l 2}\right) / 2 f_{l 1} \approx 3.8$ gives an estimation of the vortex shedding cycles in one flip-over period. The spectrum of the displacement shows the same characteristics of the lift. However, the frequency at $f_{d}=0.015$ dominates the drag spectrum with the nearby low-frequency spikes. By comparing the spectra of the drag, lift and displacement, it is seen that all results show significant peaks at 0.191 and 0.147 , indicating how the drag and the lift have the same dominant frequencies caused by the vortex shedding. This differs from the VIV of an isolated cylinder in which the drag frequency doubles the lift frequency. Note that the lower frequencies are not directly caused by the vortex shedding but the proximity interference. 
Although FFT spectral plots show overall frequency features averaged in a long time, they might not capture a temporal variation of frequencies. To understand this aspect, the Wavelet Transform (WT), time and frequency localized, has been extensively used by many researchers (Alam et al., 2003; Alam and Sakamoto, 2005; Zhao et al., 2012; Chen et al., 2015a, b). In this paper, WT of fluid forces and displacement is carried out by adopting the most commonly used complex-valued Morlet wavelet. The non-dimensional frequency was set as 6 to avoid using the correction terms (Farge, 1992). A set of discrete scales, which are evenly distributed in the frequency range obtained in the FFT analysis, were adopted.

A real part of WT is shown in Figure 7 in which colors indicate magnitudes of fluctuating amplitudes. It is observed that not only the lift amplitude shows the FF-like temporal variations, but the lift frequency also displays the same tendency, see Figures 7(bii) and 7(biii). For example, within $1510<t U_{\infty} / D<1548$, the lift on the lower cylinder (with a shorter wake) has a higher frequency $\left(f_{l 3}\right.$ $=0.191$ ) and larger vibration amplitude. Meanwhile, the lift on the upper cylinder (with a longer wake) has a lower frequency $\left(f_{12}=0.147\right)$ and smaller vibration amplitude. However, the situation flips over in the range of $1548<t U_{\infty} / D<1562$. Components at $f_{d}=0.044$ and 0.015 are clearly shown in the drag but they are hardly recognized in the lift and the displacement.

(a)

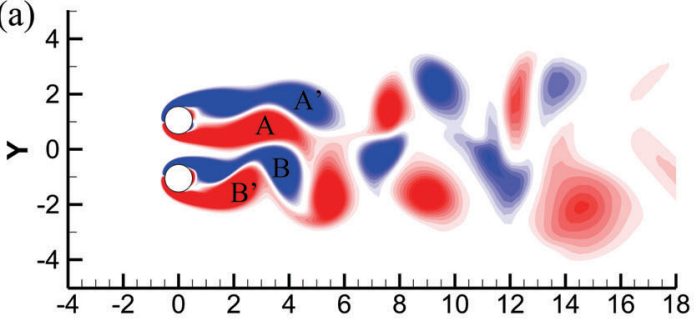

(b)

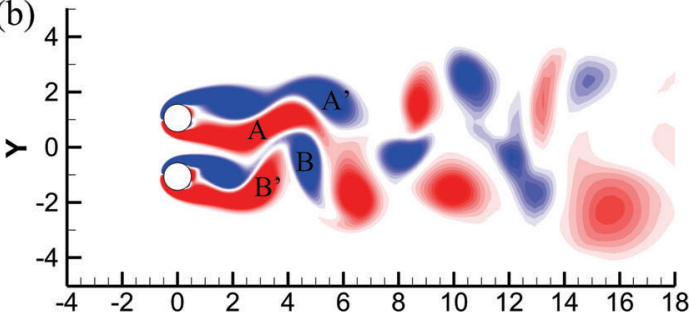

(c)

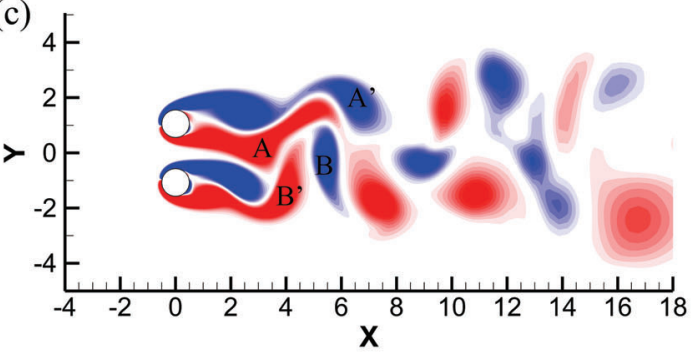

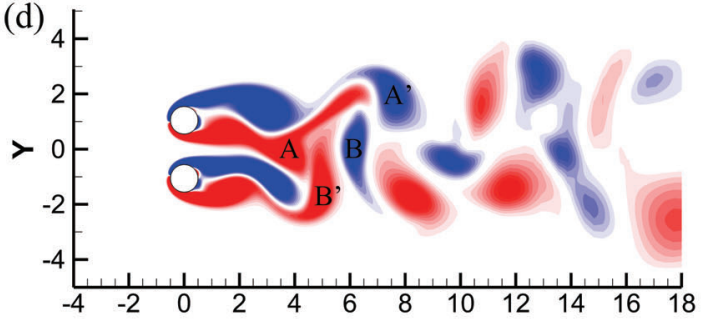

(e)

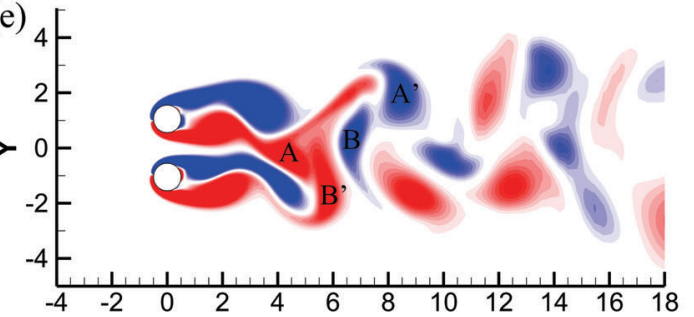

(f)

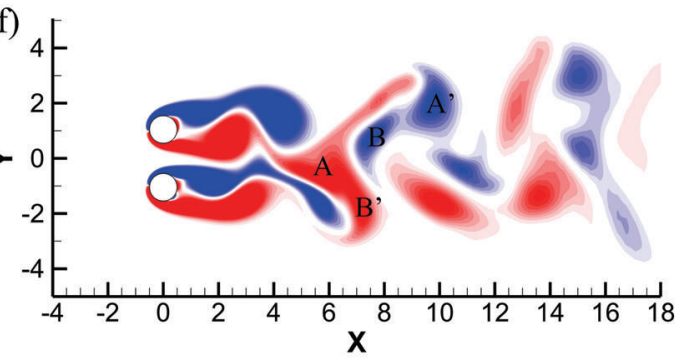

Figure 6. IFF pattern shown by vorticity contours in a vortex shedding cycle at $U_{r}=2.0$ and $s / D=$ 2.1. Instants (a) to (f) are marked by dashed vertical lines from left to right in Figure 7. 
(ai)

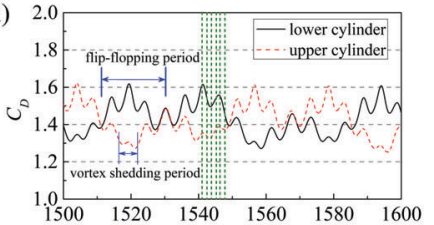

(aii)
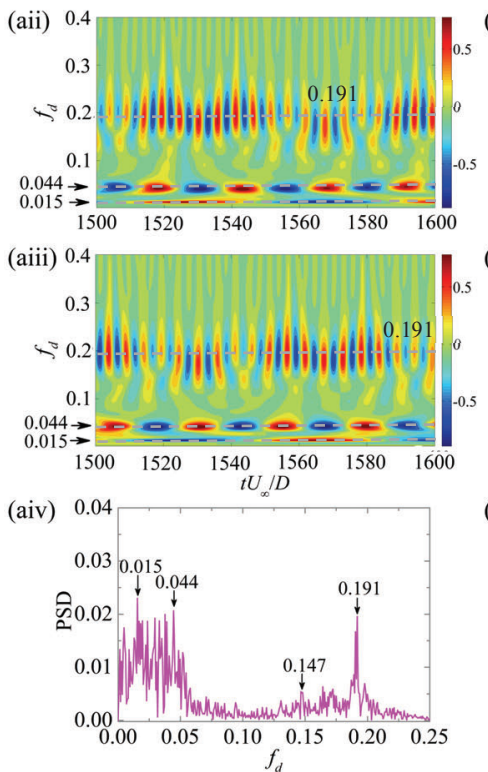
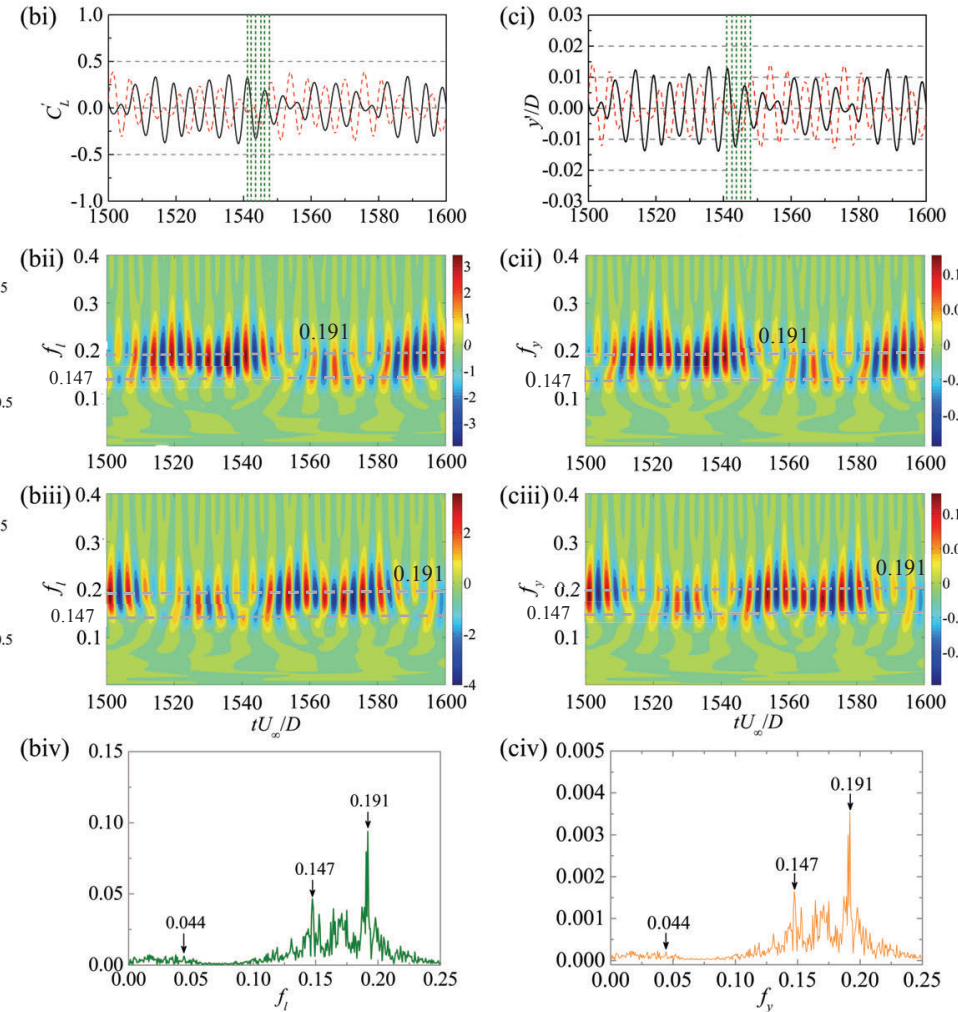
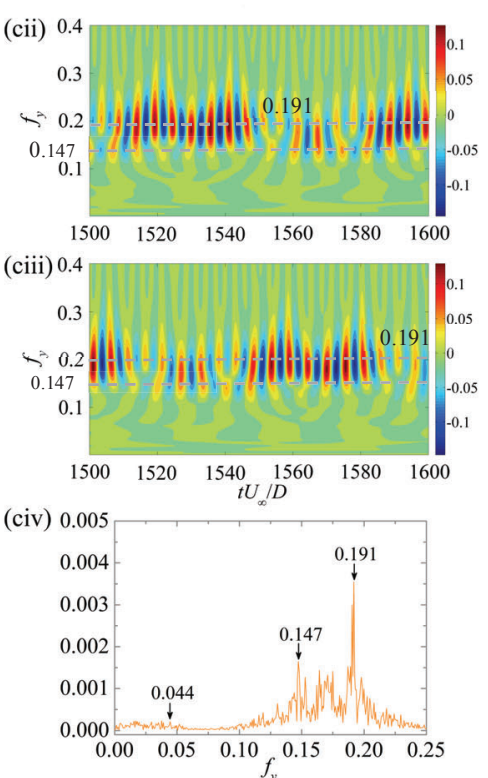

Figure 7. Time histories (i), the real part of the wavelet transform (ii, iii) and power spectral densities (iv) of the drag, lift and displacement at $U_{r}=2.0$ and $s / D=2.1$. Note that (ii) and (iii) are for lower and upper cylinders, respectively.

(a)

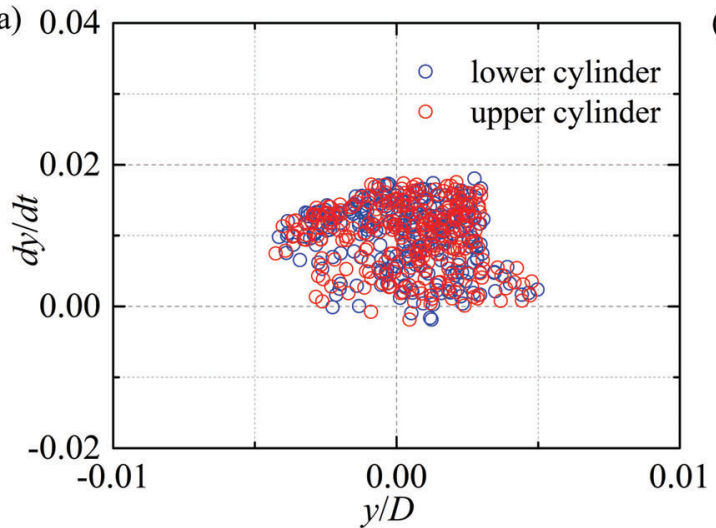

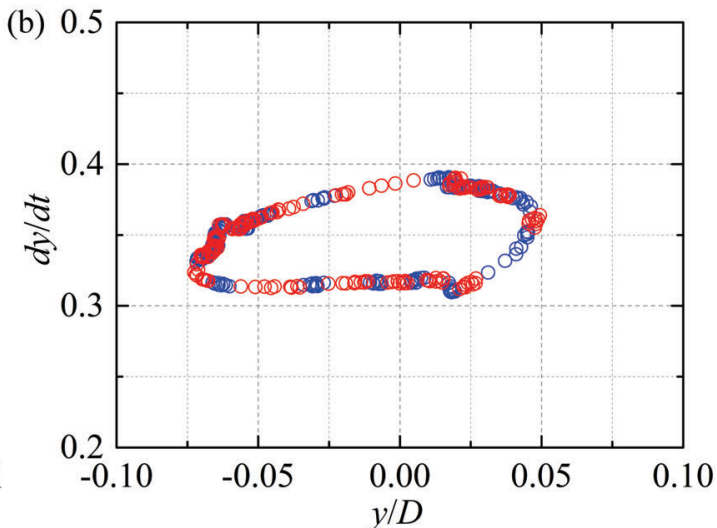

Figure 8. Poincaré maps of cylinder displacement-velocity responses: (a) IFF pattern at $U_{r}=2.0$ and $s / D=2.1$; (b) OFF pattern at $U_{r}=7.0$ and $s / D=2.4$.

\subsection{Out-of-phase flip-flopping pattern}

This OFF pattern appears within the region $5.0<U_{r}<8.0$ and $2.0<s / D<2.6$, as shown in Figure 2 . The proximity interference predominates due to a small $s / D$ and large vibration amplitude.

Figure 9 shows the vorticity contours at different instants within a vibration cycle. As the cylinders synchronously move downwards from the top position (Figures 9(a) to 9(c)), Vortex A1 shed from the inner shear layer of the upper cylinder merges with Vortex B' shed from the freestream shear layer of the lower cylinder. In the next half period, as the cylinders move upwards 
(Figures 9(d) to 9(f)), the inner shear layer of the upper cylinder shed the second vortex (Vortex A2) while the free-stream shear layer of the lower cylinder does not create the vortex shedding. A similar process occurs on vortices with the opposite sign: the free-stream shear layer of the upper cylinder creates one vortex (Vortex A') while the gap shear layer of the lower cylinder creates two vortices (Vortex B1 and B2). As shown in Figures 9(c) and 9(f), vortices pair with each other with two triplet groups, i.e. $\mathrm{A} 1+\mathrm{B}^{\prime}+\mathrm{B} 1$ and $\mathrm{B} 2+\mathrm{A}^{\prime}+\mathrm{A} 2$. This is analogous to a $2 \mathrm{~T}$ mode observed in VIV at a high $\operatorname{Re}$ (Williamson and Jauvtis, 2004). Note that the vortex shedding of the free-stream shear layer and that of the gap shear layer are not in-phase, with Vortex B' (A') leading Vortex A1 (B2). Unlike IFF pattern showing the asymmetrical vortex connections, OFF pattern displays a symmetrical vortex topology although vortices may show a slight difference in shape and size. For instance, Vortex A' is thinner and longer than Vortex B'.

Shown in Figure 10 are time histories of the drag, lift and displacement, together with WT and FFT analysis results at $U_{r}=7.0$ and $s / D=2.4$. An important observation lies in that the drag, lift and displacement have the same frequency, and they show the in-phase envelopes in IFF pattern whereas the drag, lift and displacement are out-of-phase in OFF pattern. This can be proved by the spectral plots in which the drag is dominated by a component at $f_{d}=0.285$, the lift is prevailed by a component at $f_{l}=0.210$, and the displacement is controlled by a component at $f_{y}=0.143$. The drag has a doubled dominant frequency of displacement. However, the lift dominant frequency is 1.5 times that of the displacement, which is attributed to the double vortex shedding of the inner shear layers. In addition to the dominant frequencies, the drag and lift spectra also show other peaks. The peak at $f_{d}=0.210$ in the drag spectrum and the peak at $f_{l}=0.285$ in the lift spectrum reflect the interinfluence of drag and lift forces. The peaks at $f_{d}\left(f_{i}\right)=0.068$ and 0.075 are beating-type frequencies. Moreover, the drag also contains a displacement frequency component at $f_{d}=0.143$, manifesting the FSI effect. In contrast to the multiple-peak spectra of drag and lift, the spectrum of the displacement only shows one significant peak with small neighboring spikes.

The WT analysis shows a temporal variation of different frequency contents as shown in Figure 10. It is observed that the dominant drag components on the two cylinders at $f_{d}=0.285$ are quasisynchronized with obvious frequency variations owing to the intermittent participation of the component at $f_{d}=0.353$. This is supported by the Poincaré maps of the two cylinders' trajectories in Figure 8(b). Envelopes of the dominant drag component vary in an anti-phase mode - the reason for the FF-like drag. However, the drag components on the two cylinders at $f_{d}=0.143$ and 0.068 are in anti-phase without an obvious variation. For the lift, temporal variations of the dominant components at $f_{l}=0.210$ and the associated envelops are in-phase. Frequency variations are also observed due to the existence of the component at $f_{l}=0.285$ and its intermittent characteristics. The components at $f_{l}=0.075$ are in anti-phase with an evident change in strength. The cylinder displacements reveal a mono-frequency feature with invariant spectral intensity. 

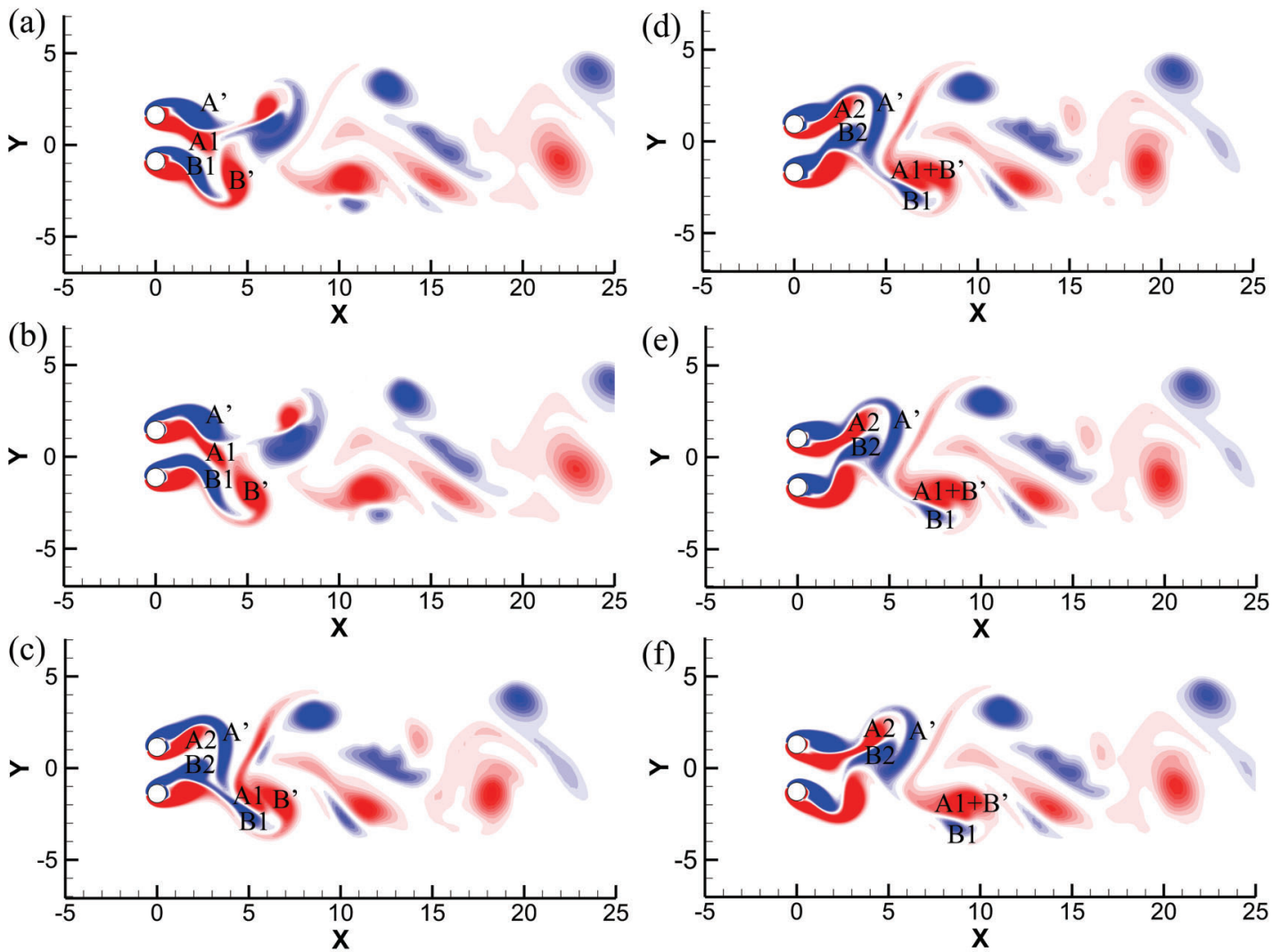

Figure 9. OFF pattern shown by vorticity contours in a vortex shedding cycle at $U_{r}=7.0$ and $s / D=$ 2.4. Instants (a) to (f) are marked by dashed vertical lines from left to right in Figure 10.
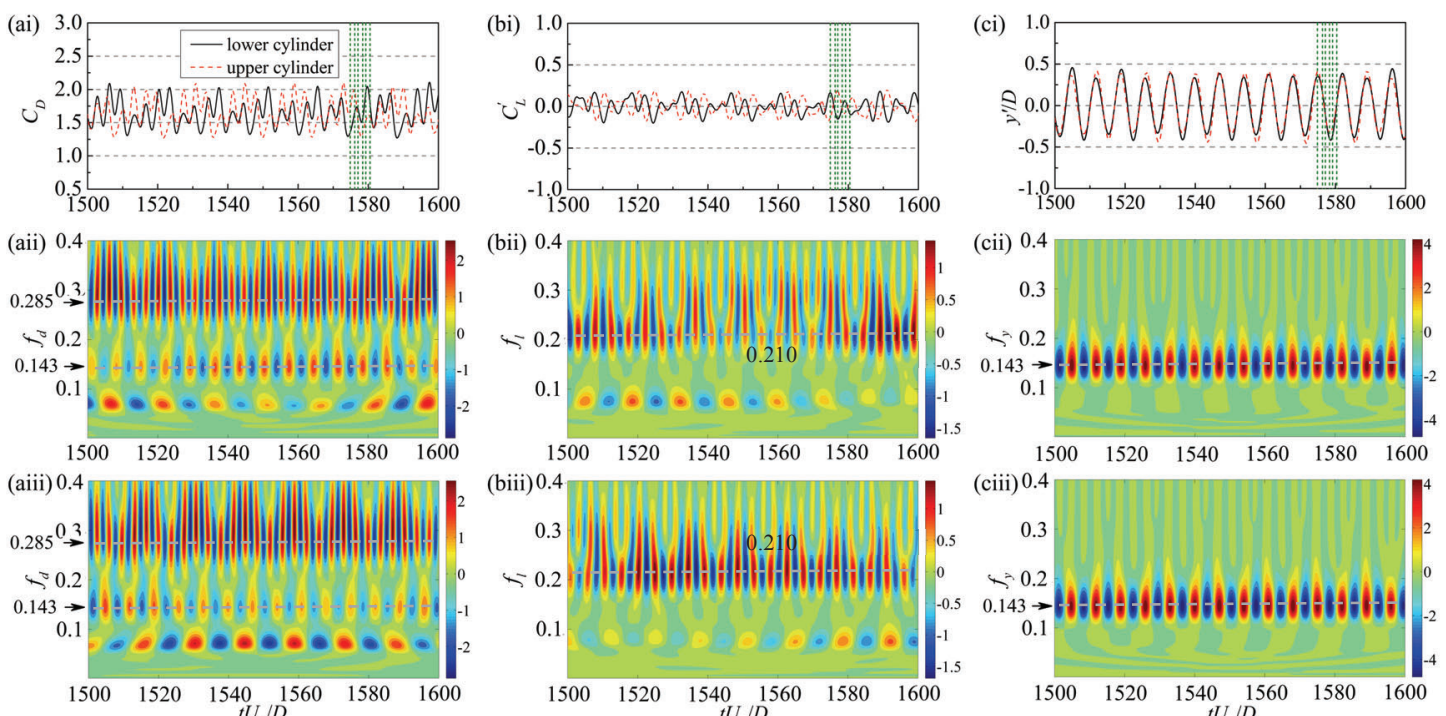

(aiv)
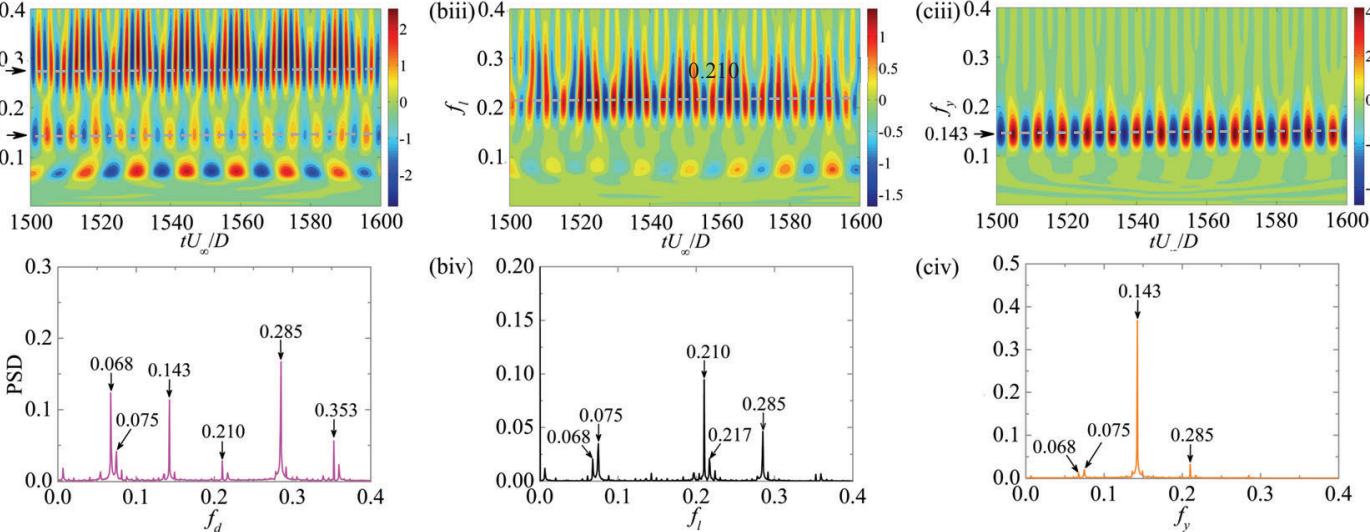

Figure 10. Time histories (i), the real part of the wavelet transform (ii, iii), and power spectral densities (iv) of the drag, lift, and displacement at $U_{r}=7.0$ and $s / D=2.4$. Note that (ii) and (iii) are for lower and upper cylinders, respectively. 


\subsection{Irregular pattern}

This IR pattern is present in the narrow region with 2.7-3.3 $<U_{r}<3.5-3.7$, corresponding to the initial branch, as shown in Figure 2. The width of the IR region varies slightly with $s / D$, and the case with $U_{r}=3.1$ and $s / D=2.5$ is selected to elaborate IR features. Figure 12 shows time histories of the drag, lift and displacement, as well as the normalized vorticity contours. It can be observed that variations of the fluid forces and the displacement are irregular and aperiodic, suggesting the unstable FSI between the cylinders. In addition, the vibration of the two cylinders is generally antiphase. gradual transition between these two patterns is observed and the border is not clearly defined.

Shown in Figure 11(a) are time histories of the drag, lift and displacement at $U_{r}=3.8$ and $s / D$ $=5.0$. The FF-like temporal variations are observed in the drag while the long-period beatings are detected in the lift and displacement. Envelopes of the drag, lift and displacement are generally inphase. Figure 11(b) shows the wake behind the cylinders at different instants marked by vertical dashed lines in Figure 11(a). In Figure 11(bi), it is seen that when the lower cylinder has a larger vibration amplitude, vortices shed from the lower cylinder are arranged in two parallel rows, showing a $\mathrm{C}(2 \mathrm{~S})$ mode. However, an alternate vortex street is observed behind the upper cylinder with a smaller amplitude, showing a $2 \mathrm{~S}$ mode. The parallel vortex street has a larger wake width and a shorter vortex formation length than that of the alternate one, resulting in a larger drag and lift and a higher vibration frequency of the lower cylinder than that of the upper cylinder. However, this wake pattern flips over in Figure 11(bii) after a non-dimensional time interval of $t U_{\infty} / D=120$, featuring the long period flip-flopping pattern.

Figure 11(c) shows the lift spectrum of the two cylinders. The highest peak exists at $f_{l}=0.1819$ with four comparable peaks on the right. An obvious low-frequency peak at $f_{l}=0.0083$, denoting the beating and flip-flopping frequency, is observed. The number of the vortex shedding in one FF period can be calculated as the ratio of the two frequencies being about 21.9. Note that the FF period of LFF pattern varies with $s / D$ and $U_{r}$. Generally, with decreasing $s / D$, the proximity interference between the cylinders becomes intensified (Le Gal et al., 1994; Peschard and Le Gal, 1996). Hence, the FF period decreases.

3
4
5
6
7
8
9
0

\subsection{Long period flip-flopping pattern}

This LFF pattern exists in a long stripe with $s / D>3.0$ and $3.6<U_{r}<4.0$ where the vibration 
(ai)

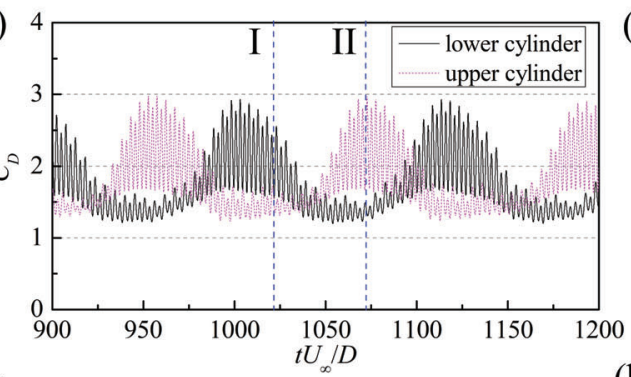

(aii)

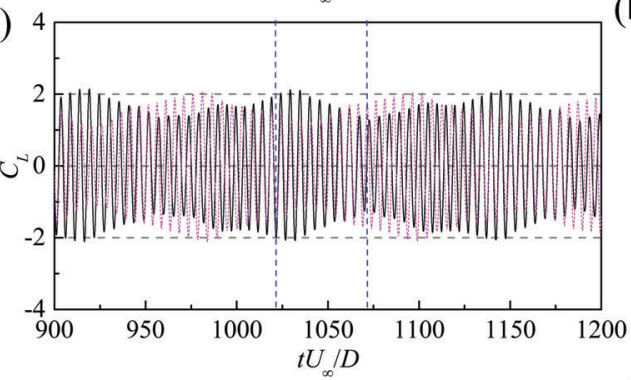

(bii) 10
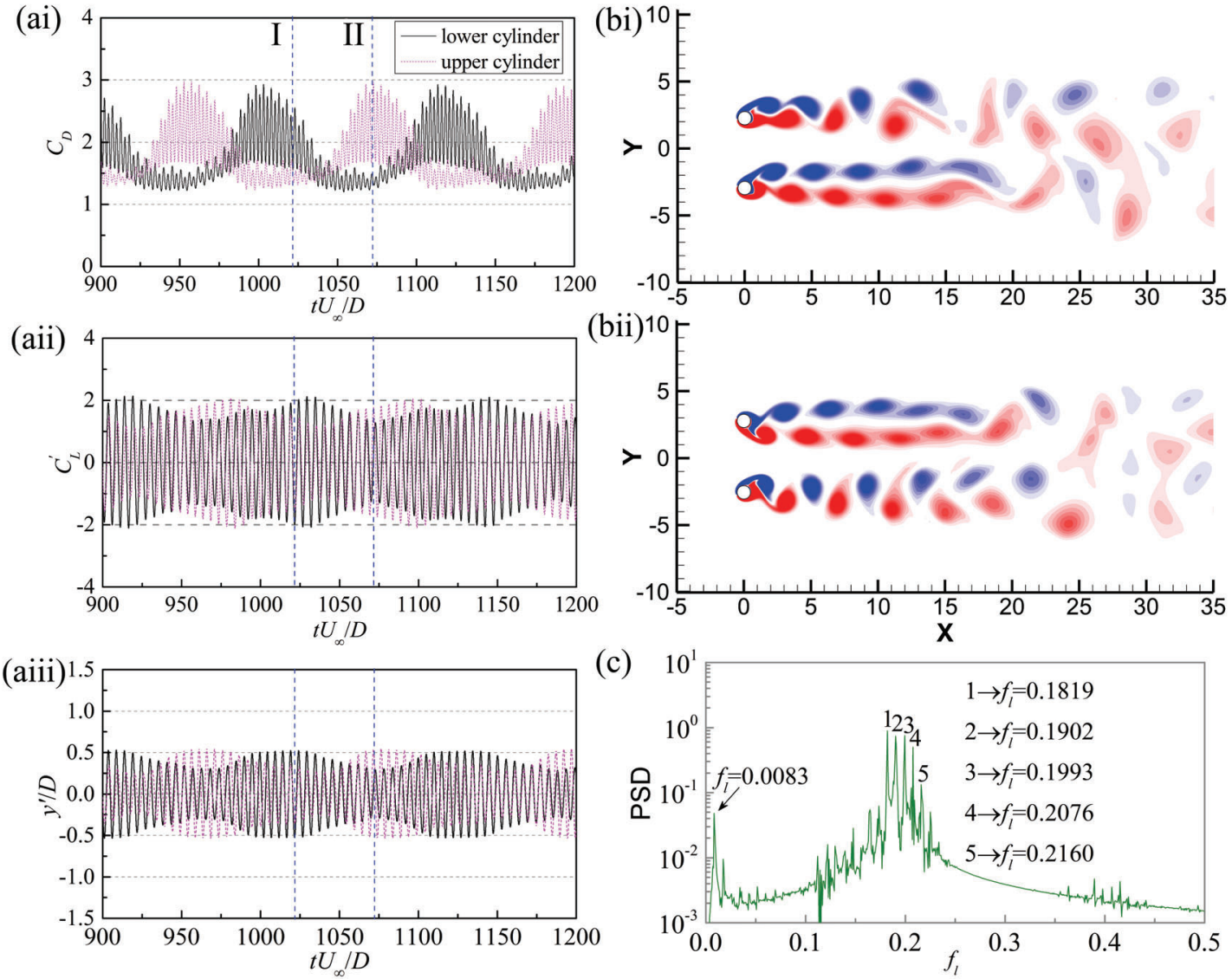

Figure 11. Time histories of drag, lift and displacement (a), vorticity contours in LFF pattern (b), and lift spectrum (c) at $U_{r}=3.8$ and $s / D=5.0$. Instants (I, II) correspond to vorticity contours in sub-plot (b).

(a)

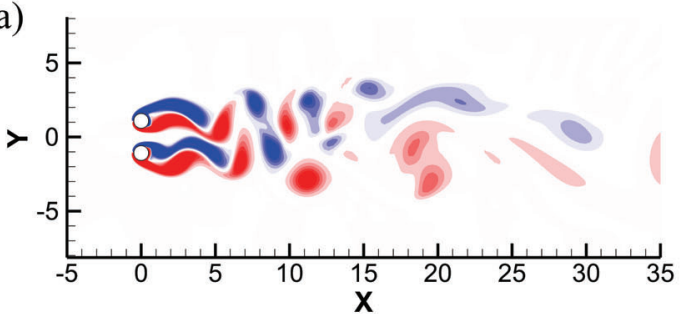

(b)

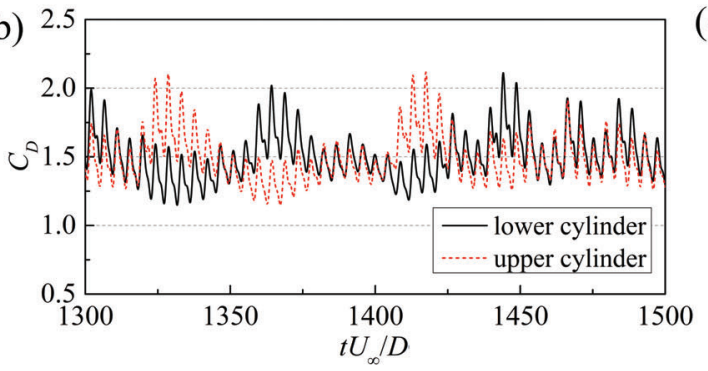

(c)

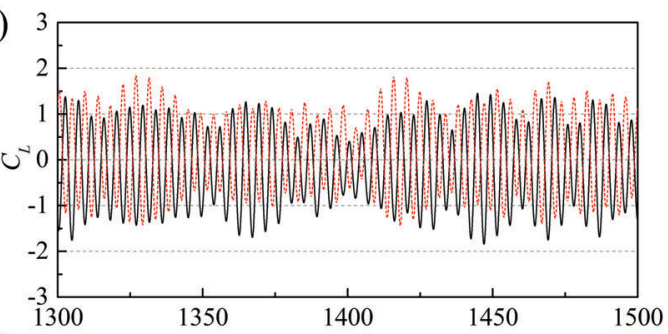

(d) 0.8

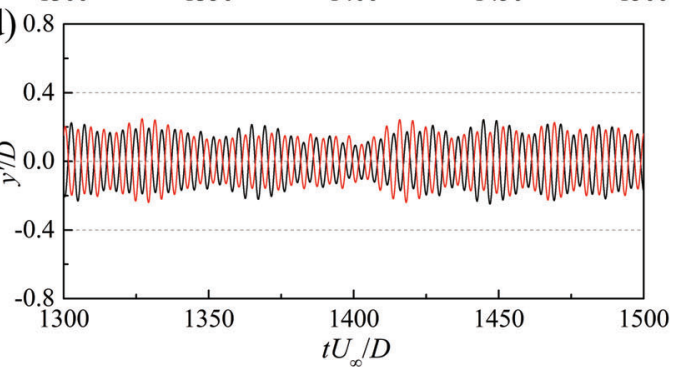

Figure 12. IR pattern (a) and time histories of drag (b), lift (c) and displacement (d) for VIV of two side-by-side circular cylinders at $U_{r}=3.1$ and $s / D=2.5$.

\subsection{Hybrid pattern}

Combining several wake characteristics, this HB pattern is observed in the range of $s / D<2.6$. 
Shown in Figure 13 are time histories of the drag, lift and displacement at $U_{r}=8.0$ and $s / D=2.2$. It is seen in the whole range that the vibration response changes between OFF and AS patterns with a transition. For example, OFF pattern occurs when $1050<t U_{\infty} / D<1104$ and $1230<t U_{\infty} / D<1300$ while AS pattern appears when $1120<t U_{\infty} / D<1170$. The two patterns compete each other in an unsteady manner, with neither one being clearly dominant. The difference between HB and IR patterns is due to a quasi-periodic appearance which is only observed in HB pattern.

(a) 2.5
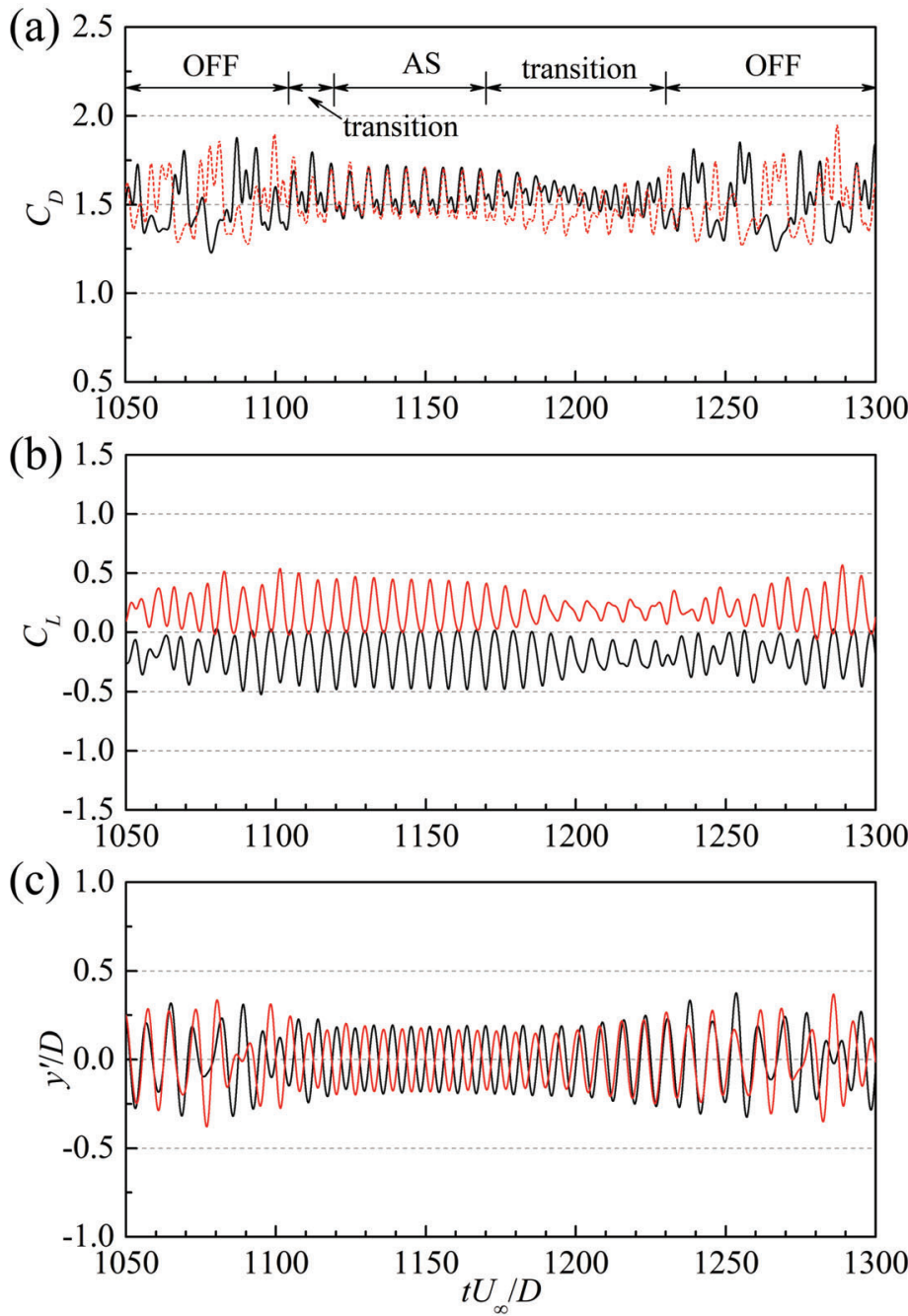

Figure 13. Time histories of drag (a), lift (b) and displacement (c) for VIV of two side-by-side circular cylinders at $U_{r}=8.0$ and $s / D=2.2$.

\section{Conclusions}

Wake patterns behind two freely vibrating side-by-side circular cylinders in laminar flows have been numerically and comprehensively investigated through the reduced velocity-cylinder spacing $\left(U_{r}, s / D\right)$ parametric space. The fixed Reynolds number is 100 , the low mass ratio is $m^{*}=2$, the spacing ratio range is $2 \leq s / D \leq 5$, and the reduced velocity range is $0 \leq U_{r} \leq 30$. The two cylinders of an equal diameter can only vibrate in the transverse direction. The parametrically specified increments are $\Delta U_{r}=0.1$ and $\Delta s / D=0.1$, achieving more than 1500 simulation case studies. 
Eight distinctive wake patterns have been identified including (i) the anti-phase synchronized pattern (AS), (ii) the in-phase synchronized pattern (IS), (iii) the biased anti-phase synchronized pattern (BAS), (iv) the in-phase flip-flopping pattern (IFF), (v) the out-of-phase flip-flopping pattern (OFF), (vi) the long period flip-flopping pattern (LFF), (vii) the irregular pattern (IR), and (viii) the hybrid pattern (HB). These are summarized in Table 3. A partition map of different wake patterns is presented with well-defined boundaries in Figure 2. In this representative map, AS pattern occupies a top portion $(s / D>3.5)$ with an exception in the range of 2.7-3.3 $<U_{r}<3.5-3.8$ where IR and LFF patterns exist. IS pattern is noticed in three sub-regions, taking the second largest portion in the $\left(U_{r}\right.$, $s / D)$ parametric plane. BAS pattern is observed in a small range of 3.9-4.0 $<U_{r}<4.5-4.8$ and $2.3<$ $s / D<2.5$. IFF pattern exists in a region of $0<U_{r}<2.7-2.9$ and $2.0<s / D<2.3$ as well as in a narrow stripe at $U_{r}=3.0$ with $2.5<S / D<3.5$. OFF pattern appears within the region $5.0<U_{r}<8.0$ and 2.0 $<s / D<2.6$. HB pattern is observed when $s / D<2$.6. An interesting hysteresis phenomenon - where the wake pattern switches when increasing or decreasing $U_{r}$ - is found between BAS/IS regions and between IS/AS regions.

Wake pattern characteristics have also been elaborated by focusing on IFF, OFF and LFF patterns. It is found that IFF pattern is featured by the long-short wake and asymmetric vortex connections. Temporal variations of the drag, lift and displacement envelopes are in-phase synchronized. On the contrary, the drag, lift and displacement are out-of-phase in OFF pattern. This can be attributed to the double vortex shedding of the inner-side shear layers within the vibration cycle. LFF pattern is characterized by a much longer flip-over interval than IFF and OFF patterns, owing to a weaker proximity interference effect between the two cylinders.

The present analyses of wake pattern characteristics in laminar flows at a low Re provide some fundamental understanding which could be useful for further studies on turbulent flows at a higher Re. Such a low-Re VIV may be encountered in an engineering practice such as a nuclear industry where Re is based on a small diameter of the heat exchange tube. Due to a large length-to-diameter ratio of the tube array, large-amplitude VIV may be excited, leading to a tube fatigue damage. This study provides physical insights into the complex interactions of the two side-by-side cylinders. Future studies will include the freely vibrating side-by-side cylinders in a higher Re and subcritical flow range, the effects of mass ratio, in-line motion and three-dimensionality.

\section{Acknowledgments}

This work was financially supported by the Science Fund for Creative Research Groups of the National Natural Science Foundation of China (Grant No. 51621092), the National Natural Science Foundation of China (Grants No. 51579175 and 51779172). Numerical simulations were performed on TianHe-1(A) at National Supercomputer Center in Tianjin. 


\section{References}

Alam, M.M., Moriya, M., Sakamoto, H., 2003. Aerodynamic characteristics of two side-by-side circular cylinders and application of wavelet analysis on the switching phenomenon. J. Fluids Struct. 18, 325-346.

Alam, M.M., Sakamoto, H., 2005. Investigation of Strouhal frequencies of two staggered bluff bodies and detection of multistable flow by wavelets. J. Fluids Struct. 20, 425-449.

Bearman, P.W., Wadcock, A.J., 1973. The interaction between a pair of circular cylinders normal to a stream. J. Fluid Mech. 61, 499-511.

Chen, W., Ji, C., Xu, W., Liu, S., Campbell, J. 2015a. Response and wake patterns of two side-byside elastically supported circular cylinders in uniform laminar cross-flow. J. Fluids Struct. 55, 218236

Chen, W., Ji, C., Wang, R., Xu, D., Campbell, J., 2015b. Flow-induced vibrations of two side-byside circular cylinders: on asymmetric vibration and symmetry hysteresis and near-wake patterns. Ocean Eng. 110, 244-257.

Chen, W., Ji, C., Williams, J., Xu, D., Yang, L., Cui, Y., 2018. Vortex-induced vibrations of three tandem cylinders in laminar cross-flow: Vibration response and galloping mechanism. J. Fluids Struct. 78, 215-238.

Cui, Z.D., Zhao, M., Teng, B., 2014. Vortex-induced vibration of two elastically coupled cylinders in side-by-side arrangement. J. Fluids Struct. 44, 270-291.

Farge, M., 1992. Wavelet transforms and their applications to turbulence. Annu. Rev. Fluid Mech. 24, 395-457.

Ji, C., Munjiza, A., Williams, J.J.R., 2012. A novel iterative direct-forcing immersed boundary method and its finite volume applications. J. Comput. Phys. 231, 1797-1821.

Kang, S., 2003. Characteristics of flow over two circular cylinders in a side-by-side arrangement at low Reynolds numbers. Phys. Fluids 15, 2486-2498.

Kim, H.J., Durbin, P.A., 1988. Investigation of the flow between a pair of circular beams in the flopping regime. J. Fluid Mech. 196, 431-448.

Le Gal, P., Chauve, M.P., Peschard, I., Jarre, S., 1994. The Ginzburg-Landau equation and the transition to turbulence in open flows. Current Topics in the Physics of Fluids 1, 307-318.

Liu, K., Ma D.-J., Sun, D.-J., Yin, X.-Y., 2007. Wake patterns of flow past a pair of circular cylinders in side-by-side arrangements at low Reynolds numbers. J. Hydrodyn. Ser. B 19(6), 690-697.

Liu, Y., So, R.M.C., Lau, Y.L., Zhou, Y., 2001. Numerical studies of two side-by-side elastic cylinders in a cross-flow. J. Fluids Struct. 15, 1009-1030.

Peschard, I., Le Gal, P., 1996. Coupled wakes of cylinders. Phys. Rev. Lett. 77, 3122-3125.

Peskin, C.S., 1972. Flow patterns around heart valves: a digital computer method for solving the equations of motion. Ph.D. thesis, Yeshiva University, New York.

Prasanth, T.K., Mittal, S., 2008. Vortex-induced vibrations of a circular cylinder at low Reynolds numbers. J. Fluid Mech. 594, 463-491. 
Sumner, D., Wong, S.S.T., Price, S.J., Païdoussis, M.P., 1999. Fluid behavior of side-by-side circular cylinders in steady cross-flow. J. Fluids Struct. 13, 309-339.

Sumner, D., 2010. Two circular cylinders in cross-flow: A review. J. Fluids Struct. 26, 849-899.

Supradeepan, K., Roy, A., 2014. Characterisation and analysis of flow over two side by side cylinders for different gaps at low Reynolds number: A numerical approach. Phys. Fluids 26, 063602 .

Williamson, C.H.K., 1985. Evolution of a single wake behind a pair of bluff bodies. J. Fluid Mech. 159, 1-18.

Williamson, C.H.K., Jauvtis, N., 2004. A high-amplitude $2 t$ mode of vortex-induced vibration for a light body in XY motion. Eur. J. Mech. B-Fluids, 23, 107- 114.

Williamson, C.H.K., Govardhan, R., 2004. Vortex-induced vibration. Annu. Rev. Fluid Mech. 36, 413-455.

Xu, Y.S., Liu, Y., Xia, Y., Wu, F.M., 2008. Lattice-Boltzmann simulation of two-dimensional flow over two vibrating side-by-side circular cylinders. Phys. Rev. E 78, 046314.

Zdravkovich, M.M., 1977. Review of flow interference between two circular cylinders in various arrangements. Trans. ASME: J. Fluids Engng 99, 618-633.

Zdravkovich, M.M., 1997. Flow Around Circular Cylinders, vol 1: Fundamentals. Oxford University Press.

Zhao, M., Cheng, L., An, H.W., 2012. Numerical investigation of vortex-induced vibration of a circular cylinder in transverse direction in oscillatory flow. Ocean Engng 41, 39-52.

Zhou, Y., Alam, M.M., 2016. Wake of two interacting circular cylinders: a review. Int. J. Heat Fluid Flow 62, 510-537. 


\section{Highlights}

- Wake patterns of two transversely vibrating side-by-side circular cylinders are numerically investigated.

- Cylinders with low mass ratio of 2 are subject to uniform laminar flows with $\operatorname{Re}=100$.

- Cylinder spacing ratio is varied between 2 and 5 with small increment of 0.1 .

- Reduced flow velocity is varied between 0 and 30 with small increment of 0.1.

- Over 1500 new simulation cases are characterized into 8 distinctive wake patterns. 